\title{
Left Ventricular Abnormality and Covert Atrial Fibrillation in Embolic Stroke of Undetermined Source
}

\author{
Hajime Ikenouchi ${ }^{1}$, Junpei Koge ${ }^{1}$, Tomotaka Tanaka ${ }^{2}$, Eriko Yamaguchi ${ }^{1}$, Shuhei Egashira ${ }^{1}$, Kazuo Washida ${ }^{2}$, \\ Satoshi Nagase ${ }^{3}$, Kengo Kusano ${ }^{4}$, Kazunori Toyoda ${ }^{1}$, Masafumi Ihara ${ }^{2}$ and Masatoshi Koga ${ }^{1}$
${ }^{1}$ Department of Cerebrovascular Medicine, National Cerebral and Cardiovascular Center, Suita, Japan
${ }^{2}$ Department of Neurology, National Cerebral and Cardiovascular Center, Suita, Japan
${ }^{3}$ Department of Advanced Arrhythmia and Translational Medical Science, National Cerebral and Cardiovascular Center, Suita, Japan
${ }^{4}$ Department of Cardiovascular Medicine, National Cerebral and Cardiovascular Center, Suita, Japan

Aims: The relationship between left ventricular (LV) function and AF detection in embolic stroke of undetermined source (ESUS) patients with insertable cardiac monitors (ICMs) remains unclear. We investigated the association between LV function and AF detection in patients with ESUS after ICMs implantation.

Methods: We enrolled patients with ESUS who underwent ICMs implantation from September 2016 to September 2020 using a single-center, prospective registry. LV systolic and diastolic functions were assessed on precordial echocardiography by LV fractional shortening (LVFS) and average E/e', respectively. Associations between characteristics of LV function and detection of AF by ICMs were analyzed.

Results: Participants comprised 101 patients (median age, 74 years; male, 62\%). During a median follow-up period of 442 days (interquartile range (IQR), 202-770 days), AF was detected in 24 patients (24\%). Median duration from ICMs implantation to AF detection was 71 days (IQR, 13-150 days). When LVFS and E/e' were dichotomized by cutoff value, each of low LVFS ( $<35.5 \%$; adjusted hazard ratio (HR), 4.77; 95\% confidence interval (CI), 1.77-12.9) and high E/e' ( $\geq 8.65$; adjusted HR, 4.56; 95\% CI, 1.17-17.7) were independently associated with AF detection after adjusting for age and sex. When patients were divided into four groups according to dichotomized LVFS and E/e', the combination of low LVFS and high E/e' was independently associated with $\mathrm{AF}$.

Conclusions: In patients with ESUS after ICMs implantation, the LV characteristics of low LVFS and high E/e' were associated with AF detection.

Key words: Ischemic stroke, Left ventricular function, Left ventricular hypertrophy, Insertable cardiac monitor, Embolic stroke of undetermined source

\section{Introduction and Aims}

Insertable cardiac monitors (ICMs) are useful to detect covert atrial fibrillation (AF) in patients with embolic stroke of undetermined source (ESUS) who could not be detected AF by usual electrocardiogram monitoring ${ }^{1)}$. Since ICMs implantation is invasive and costly, we have to select candidates for ICMs implantation among ESUS patients. Strong predictors for AF detection in ESUS patients would be clinically relevant. Regarding the pathophysiology of the development of AF, left atrial (LA) remodeling has been known as one of the major contributors ${ }^{2}$. The left ventricular (LV) systolic and diastolic dysfunction has been reported to contribute to the remodeling of the LA, suggesting the LV dysfunction may also be important predictors of $\mathrm{AF}^{3-5)}$. Actually, LV dysfunction has been associated with $\mathrm{AF}$ in the previous population-based study ${ }^{6}$. In terms of $\mathrm{AF}$ detection in ESUS patients by ICMs, factors of the

Address for correspondence: Junpei Koge, Department of Cerebrovascular Medicine, National Cerebral and Cardiovascular Center, 6-1 Kishibe-shimmachi, Suita, Osaka 564-8565, Japan E-mail: koge.jumpei@ncvc.go.jp

Received: April 26, $2021 \quad$ Accepted for publication: June 28, 2021

Copyright@2021 Japan Atherosclerosis Society

This article is distributed under the terms of the latest version of CC BY-NC-SA defined by the Creative Commons Attribution License. 
LA remodeling such as the LA enlargement and frequent premature atrial contraction $(\mathrm{PAC})^{7}$ have reportedly been associated with AF detection ${ }^{8,9)}$. However, no studies focused on the relationship between LV dysfunction and AF detection by ICM in ESUS patients. Consequently, the predictive value of the LV dysfunction for AF detection in these cohorts remains to be elucidated. The present study aimed to investigate the association between the LV dysfunction and AF detection using the cohort of ESUS with ICMs implantation.

\section{Methods}

\section{Study Population}

All patients with ESUS and ICMs implantation were prospectively registered to the ICMs registry at the National Cerebral and Cardiovascular Center (NCVC). Patients registered from September 2016 to October 2020 were retrospectively reviewed. Inclusion criteria for the ICMs registry were patients diagnosed with ESUS. ESUS was diagnosed based on the criteria by the Cryptogenic Stroke/ESUS International Working Group ${ }^{10)}$. ICMs were implanted according to the recommendations in the Japanese clinical guide, which recommended brain magnetic resonance imaging (MRI), long-term cardiac monitoring including Holter ECG, assessment of extra- and intracranial arteries supplying the area of brain ischemia as mandatory examinations, and strongly recommended transesophageal echocardiography and ultrasonographic examinations for right-to-left shunt or aortic $\mathrm{CT}^{11)}$. This ICMs registry was approved by the institutional review board of NCVC (approval number: R20087). The opt-out method for patient recruitment was used.

\section{Clinical Data Collection}

We collected the following clinical demographic data and comorbidities from medical records: age, sex, hypertension (diagnosis at hospital discharge or use of antihypertensive medications), dyslipidemia (diagnosis at hospital discharge or use of statins), diabetes mellitus (diagnosis at hospital discharge or use of oral hypoglycemic agents or insulin), $\mathrm{CHA}_{2} \mathrm{DS}_{2}$-VASc score after index stroke and baseline National Institutes of Health Stroke Scale (NIHSS) score. Concentration of BNP and estimated glomerular filtration rate ${ }^{12)}$ were collected from blood tests. The PAC rate was calculated by the total number of PACs per day of 24-h Holter electrocardiogram monitoring. LV fractional shortening (LVFS), left atrial diameter, average E/e' and presence of LV hypertrophy were evaluated by transthoracic echocardiography. LV systolic function was assessed by LVFS. LVFS was calculated according to the formula: fractional shortening $(\mathrm{FS})=($ LVIDd - LVIDs $) \quad / \quad$ LVIDd $\times 100$ (\%), where LVIDd indicates the LV internal dimension at end-diastole and LVIDs indicates the LV internal dimension at end-systole ${ }^{13)}$. LV diastolic function was assessed as the average E/e'. Doppler tissue imaging of the mitral annulus was obtained from the apical four-chamber view using a 1- to 2-mm sample volume placed in the septal and lateral mitral valve annulus. The $\mathrm{E}$ wave reflects mitral inflow and the e' wave reflects mitral annulus velocity. Average E/ $e^{\prime}$ was calculated as the average of septal E/e' and lateral E/e' values, and it is known to be accurate noninvasive predictor of increased LV filling pressure and LV diastolic dysfunction ${ }^{14)}$. LV hypertrophy was assessed from LV mass index (LVMI) indexed to the body surface area, as follows: $\mathrm{LV}$ mass $=0.8 \times 1.04$ $\left[(\text { LVIDd + LVPWTd + IVSTd })^{3}-(\text { LVIDd })^{3}\right]+0.6$, where IVSTd was the diastolic interventricular septal wall thickness, and LVPWTd was the diastolic LV posterior wall thickness. LV hypertrophy was defined as $>115 \mathrm{~g} / \mathrm{m}^{2}$ for male patients and $>95 \mathrm{~g} / \mathrm{m}^{2}$ for female patients ${ }^{15}$. Mean left atrial appendage velocity, presence of smoke-like echo and patent foramen ovale were assessed by transesophageal echocardiography.

\section{ICMs Findings and AF Detection}

ICMs (Reveal LINQ; Medtronic, Irvine, CA, USA, and Biomonitor III; Biotronic, Berlin, Germany) were implanted under local anesthesia. These devices can automatically detect and store AF episodes $\geq 2 \mathrm{~min}$ according to the unique $\mathrm{AF}$ detection algorithm. Data transmission was remotely performed. A real-time report was obtained from each patient. A study physician received an alert if the device detected an AF episode. AF was then confirmed by an expert cardiologist (Dr. Nagase). Written informed consent was obtained for ICMs implantation and remote monitoring.

\section{Outcomes Measures}

The primary outcome of the present study was detection of AF by ICMs.

\section{Statistical Analysis}

We compared the above clinical information between patients with and without AF. Continuous variables are reported as median and interquartile range (IQR). Categorical variables are presented as frequency and proportion. Continuous variables were assessed using the Wilcoxon rank-sum test. Categorical variables were analyzed using the 2-sided Fisher's exact test. For univariate analyses, we compared baseline 
Table 1. Baseline clinical characteristics according to detection of AF

\begin{tabular}{|c|c|c|c|c|}
\hline & All $(n=101)$ & $\mathrm{AF}-(n=77)$ & $\mathrm{AF}+(n=24)$ & $p$-value \\
\hline Male & $62(61)$ & $47(61)$ & $15(63)$ & 1.00 \\
\hline Age, years & $74(62-79)$ & $72(60-77)$ & $78(70-81)$ & 0.02 \\
\hline Hypertension & $69(68)$ & $53(69)$ & $16(67)$ & 1.00 \\
\hline Dyslipidemia & $60(59)$ & $46(59)$ & $14(58)$ & 1.00 \\
\hline Diabetes & $14(14)$ & $9(12)$ & $5(21)$ & 0.31 \\
\hline $\mathrm{CHA}_{2} \mathrm{DS}_{2}$-VASc score & $3(1-4)$ & $3(1-4)$ & $3(2-4)$ & 0.32 \\
\hline NIHSS score & $2(0-5)$ & $1(0-3)$ & $3(1-9)$ & $<0.01$ \\
\hline \multicolumn{5}{|l|}{ Blood test results } \\
\hline BNP, pg/ml & $41(14-85)$ & $25(11-53)$ & $118(47-200)$ & $<0.01$ \\
\hline $\mathrm{eGFR}, \mathrm{ml} / \mathrm{min}$ & $64(53-77)$ & $63(52-74)$ & $67(57-82)$ & 0.23 \\
\hline \multicolumn{5}{|l|}{ 24-h Holter ECG findings } \\
\hline Rate of PAC, \% & $0.10(0.02-0.45)$ & $0.06(0.02-0.25)$ & $0.55(0.10-3.85)$ & $<0.01$ \\
\hline \multicolumn{5}{|l|}{ TTE findings } \\
\hline LVFS, \% & $36(32-41)$ & $37(33-41)$ & $33(30-35)$ & 0.01 \\
\hline Average E/e' $(n=79)$ & $8.8(7.3-11.6)$ & $8.4(7.2-10.2)$ & $11.6(8.8-17.2)$ & $<0.01$ \\
\hline $\mathrm{LAD}, \mathrm{mm}$ & $35(32-39)$ & $34(32-38)$ & $38(33-43)$ & $<0.05$ \\
\hline LV hypertrophy & $14(14)$ & $7(9)$ & $7(29)$ & 0.02 \\
\hline \multicolumn{5}{|l|}{ TEE findings } \\
\hline Mean LAA velocity, $\mathrm{cm} / \mathrm{s}$ & $60(44-76)$ & $61(50-76)$ & $51(32-79)$ & 0.11 \\
\hline Smoke-like echo & $3(3)$ & $0(0)$ & $3(13)$ & 0.01 \\
\hline Patent foramen ovale & $53(52)$ & $38(49)$ & $15(63)$ & 0.35 \\
\hline \multicolumn{5}{|l|}{ ICMs findings } \\
\hline Stroke onset to implant, days & $57(20-112)$ & $61(27-115)$ & $30(15-61)$ & 0.01 \\
\hline Duration of observation, days & $442(202-770)$ & $358(159-705)$ & $710(421-829)$ & $<0.01$ \\
\hline
\end{tabular}

Data are presented as $n(\%)$ or median (interquartile range).

Abbreviations: AF, atrial fibrillation; NIHSS, National Institutes of Health Stroke Scale; BNP, brain natriuretic peptide; eGFR, estimated glomerular filtration rate; ECG, electrocardiogram; PAC, premature atrial contraction; LVFS, left ventricle fractional shortening; LAD, left atrial diameter; LAA, left atrial appendage; ICMs, insertable cardiac monitors; TTE, transthoracic echocardiography; TEE, transesophageal echocardiography.

characteristics and physiological markers between groups dichotomized by the cutoff values of LVFS and E/e'. A receiver operating characteristic (ROC) curve analysis using the Youden Index was performed to determine the optimal cutoff value of LVFS and E/e'. Cox proportional hazard models for detection of AF were created in groups dichotomized by LVFS and E/ e'. These models were adjusted for age and sex. In addition, we compared cumulative AF-free rates among 4 groups dichotomized by LVFS and E/e' using Holm-Sidak multiple comparisons testing. We also compared the presence of LV hypertrophy among the 4 groups. Missing data were handled with pairwise deletion. Values of $p<0.05$ were considered statistically significant. The $95 \%$ confidence intervals (CIs) were calculated for all measures that required reporting of statistical uncertainty. Statistical analyses were performed in JMP version 14.3.0 (SAS Institute, Cary, NC, USA).

\section{Results}

A total of 101 patients (median age, 74 years; IQR, 62-79 years; 61\% male) were enrolled in the registry and selected as the available cohort. Reveal LINQ and Biomonitor III ICMs were implanted in 95 patients $(94 \%)$ and 6 patients $(6 \%)$, respectively. Median time from stroke onset to ICMs implantation was 57 days (IQR, 20-112 days). Median duration of observation after ICMs implantation was 442 days (IQR, 202-770 days). AF was detected in 24 patients (24\%) at a median of 71 days (IQR, 13-150 days) after ICMs implantation (Table 1). There were no patients with symptomatic heart failure. No data were missing for LVFS, but E/e' was missing for 22 patients (22\%). Patients with AF showed lower LVFS and higher average E/e' than patients without AF, in addition to higher levels of BNP, higher frequency of PACs, and larger left atrial diameter. Patients with AF also displayed a shorter interval from stroke onset to ICMs implantation and longer duration of 
Table 2. Comparison of clinical characteristics between patients with high and low LVFS, and between patients with high and low E/e'

\begin{tabular}{|c|c|c|c|c|c|c|}
\hline & $\begin{array}{l}\text { Low LVFS }<35.5 \% \\
\qquad(n=49)\end{array}$ & $\begin{array}{c}\text { High LVFS } \geq 35.5 \\
(n=52)\end{array}$ & $p$-value & $\begin{array}{c}\text { Low } \mathrm{E} / \mathrm{e}^{\prime}<8.65 \\
(n=38)\end{array}$ & $\begin{array}{l}\text { High E/e' } \geq 8.65 \\
(n=41)\end{array}$ & $p$-value \\
\hline Male & $34(69)$ & $28(54)$ & 0.15 & $33(87)$ & $18(44)$ & $<0.01$ \\
\hline Age, years & $73(61-79)$ & $75(63-79)$ & 0.49 & $63(52-76)$ & $77(73-81)$ & $<0.01$ \\
\hline Hypertension & $35(71)$ & $34(65)$ & 0.53 & $20(53)$ & $32(78)$ & 0.02 \\
\hline Dyslipidemia & $27(55)$ & $33(64)$ & 0.42 & $22(58)$ & $26(63)$ & 0.65 \\
\hline Diabetes & $8(16)$ & $6(12)$ & 0.57 & $4(11)$ & $7(17)$ & 0.52 \\
\hline $\mathrm{CHA}_{2} \mathrm{DS}_{2}$-VASc score & $3(2-4)$ & $3(1-4)$ & 0.66 & $2(1-3)$ & $3(3-5)$ & $<0.01$ \\
\hline NIHSS score & $2(0-6)$ & $1(0-3)$ & 0.43 & $1(0-4)$ & $1(0-3)$ & 0.66 \\
\hline \multicolumn{7}{|l|}{ Blood test results } \\
\hline BNP, pg/ml & $36(12-112)$ & $41(19-62)$ & 0.63 & $19(6-42)$ & $53(24-112)$ & $<0.01$ \\
\hline eGFR, $\mathrm{ml} / \mathrm{min}$ & $63(53-73)$ & $65(52-77)$ & 0.68 & $63(52-76)$ & $64(57-72)$ & 0.93 \\
\hline \multicolumn{7}{|l|}{ 24-h Holter ECG findings } \\
\hline PAC, $\%$ & $0.06(0.02-0.61)$ & $0.13(0.02-0.38)$ & 0.98 & $0.03(0.01-0.21)$ & $0.15(0.05-0.50)$ & 0.01 \\
\hline \multicolumn{7}{|l|}{ TTE findings } \\
\hline $\mathrm{LAD}, \mathrm{mm}$ & $35(32-40)$ & $35(33-38)$ & 0.84 & $34(31-36)$ & $38(33-42)$ & $<0.01$ \\
\hline LV hypertrophy & $7(14)$ & $6(12)$ & 0.77 & $0(0)$ & $9(22)$ & $<0.01$ \\
\hline \multicolumn{7}{|l|}{ TEE findings } \\
\hline Mean LAA velocity, $\mathrm{cm} / \mathrm{s}$ & $60(46-80)$ & $62(44-70)$ & 0.67 & $66(55-78)$ & $54(39-64)$ & $<0.01$ \\
\hline Smoke-like echo & $3(6)$ & $0(0)$ & 0.11 & $0(0)$ & $3(7)$ & 0.24 \\
\hline Patent foramen ovale & $28(57)$ & $25(48)$ & 0.43 & $20(53)$ & $22(54)$ & 1.00 \\
\hline \multicolumn{7}{|l|}{ ICMs findings } \\
\hline Stroke onset to implant, days & $55(19-107)$ & $58(26-111)$ & 0.77 & $45(24-121)$ & $59(27-84)$ & 0.76 \\
\hline Duration of observation, days & $526(218-801)$ & $355(185-750)$ & 0.27 & $306(103-701)$ & $397(204-758)$ & 0.23 \\
\hline AF detection & $19(39)$ & $5(10)$ & $<0.01$ & $3(8)$ & $14(34)$ & $<0.01$ \\
\hline
\end{tabular}

Data are presented as $\mathrm{n}(\%)$ or median (interquartile range).

Abbreviations: NIHSS, national institute of health stroke scale; BNP, brain natriuretic peptide; eGFR, estimated glomerular filtration rate; ECG, electrocardiogram; PAC, premature atrial contraction; LVFS, left ventricular fractional shortening; LAD, left atrial diameter; LAA, left atrial appendage; ICMs, insertable cardiac monitors; TTE, transthoracic echocardiography; TEE, transesophageal echocardiography; AF, atrial fibrillation.

Table 3. Cox proportional hazard analysis for AF detection on low LVFS and high E/e'

\begin{tabular}{|c|c|c|c|c|c|c|c|c|}
\hline & \multicolumn{2}{|l|}{ Model 1} & \multicolumn{2}{|l|}{ Model 2} & \multicolumn{2}{|l|}{ Model 3} & \multicolumn{2}{|l|}{ Model 4} \\
\hline & $\operatorname{HR}(95 \% \mathrm{CI})$ & $P$ value & $\operatorname{HR}(95 \% \mathrm{CI})$ & $P$ value & $\operatorname{HR}(95 \% \mathrm{CI})$ & $P$ value & $\operatorname{HR}(95 \% \mathrm{CI})$ & $P$ value \\
\hline Low LVFS and high E/e' & $6.65(2.32-19.1)$ & $<0.01$ & $4.44(1.47-13.4)$ & $<0.01$ & $8.73(3.21-23.8)$ & $<0.01$ & $6.84(2.22-21.1)$ & $<0.01$ \\
\hline
\end{tabular}

model 1; adjusting for age, model 2; adjusting for BNP, model 3; adjusting for PAC, model 4; adjusting for LAD

Abbreviations: AF, atrial fibrillation; HR, hazard ratio; CI, confidence interval; LVFS, left ventricular fractional shortening; BNP, brain natriuretic peptide; PAC, premature atrial contraction; LAD, left atrial diameter.

monitoring (Table 1$)$.

The area under the curve (AUC) of LVFS and E/e' was $0.67(95 \%$ CI $0.55-0.80)$ and $0.72(95 \%$ CI $0.58-0.87)$ and optimal cutoff value of LVFS and E/e' was $35.5 \%$ and 8.65 , respectively. Comparisons of clinical characteristics between patients with high/low LVFS and high/low E/e' are shown in Table 2. LVFS and E/e' were dichotomized about values of $35.5 \%$ and 8.65, respectively. On univariate analyses, low LVFS and high E/e' were each associated with higher frequencies of AF detection. Patients with high E/e' showed significantly higher BNP, larger left atrial diameter, and higher frequencies of LV hypertrophy and PACs (Table 2). Cox proportional hazard modeling adjusting for age and sex demonstrated that each of low LVFS (adjusted HR, 4.77; 95\% CI, $1.77-$ 12.9) and high E/e' (adjusted $\mathrm{HR}, 4.56$; 95\%CI, 1.17-17.7) were associated with AF as detected by the ICMs. The combination of low LVFS and high E/e' (crude HR, 9.38; 95\% CI, 3.46-25.5) was associated with AF detection even after adjusting for age, BNP, PAC or LAD (Table 3). 
Table 4. Cox proportional hazard analysis for AF detection according to groups dichotomized by LVFS and E/e'

\begin{tabular}{lcccc}
\hline & Patients, $n$ & AF, $n(\%)$ & HR (95\%CI) & $p$-value \\
\hline $\begin{array}{l}\text { Group 1 } \\
\text { Low LVFS and low E/e' }\end{array}$ & 18 & $2(11)$ & 1.00 (reference) \\
$\begin{array}{l}\text { Group 2 } \\
\quad \text { High LVFS and low E/e' }\end{array}$ & 20 & $1(5)$ & $0.42(0.04-4.68)$ & 0.48 \\
$\begin{array}{l}\text { Group 3 } \\
\quad \text { High LVFS and high E/e' }\end{array}$ & 25 & $3(12)$ & $0.97(0.16-5.81)$ & 0.97 \\
$\begin{array}{l}\text { Group 4 } \\
\text { Low LVFS and high E/e' }\end{array}$ & 16 & $11(69)$ & $7.82(1.73-35.36)$ & $<0.01$ \\
\hline
\end{tabular}

Abbreviations: AF, atrial fibrillation; HR, hazard ratio; CI, confidence interval; LVFS, left ventricular fractional shortening.

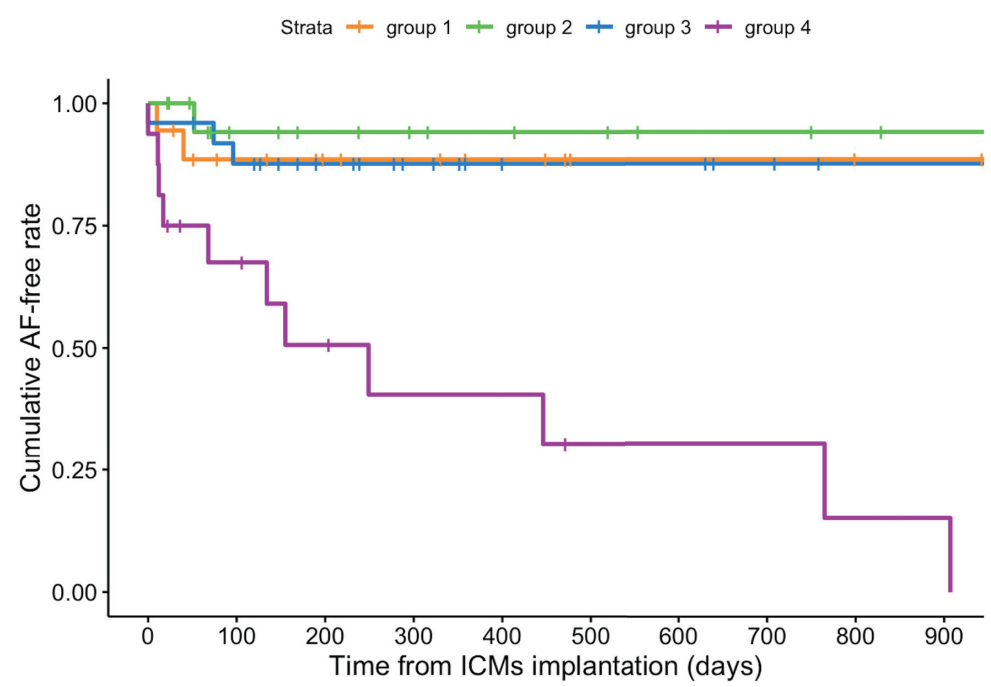

Fig. 1. Cumulative AF-free rate among groups dichotomized by LVFS and E/e'

Days from implantation of ICMs to AF detection were significantly shorter in patients with Group 4 (low LVFS and high E/e' group) than in those without on Holm-Sidak multiple comparisons test. Group 1 represents patients with low LVFS and low E/e'; Group 2 represents patients with high LVFS and low E/e'; Group 3 represents patients with high LVFS and high E/e'; Group 4 represents patients with low LVFS and high E/e'.

Abbreviations: AF, atrial fibrillation; LVFS, left ventricular fractional shortening; ICMs, insertable cardiac monitors.

We repeated Cox proportional hazard analysis and Holm-Sidak multiple comparisons testing for 4 subgroups categorized according to the combinations of dichotomized LVFS and E/e’. Group 1 comprised 18 patients with low LVFS and low E/e' and showed an AF detection rate of $11 \%$. Group 2 consisted of 20 patients with high LVFS and low E/e', with a 5\% AF detection rate. Group 3 consisted of 25 patients with high LVFS and high E/e', with a 12\% AF detection rate. Group 4 comprised 16 patients with low LVFS and high E/e', with a 69\% AF detection rate. On Cox proportional hazard analysis, only Group 4 showed a significantly higher hazard ratio for AF detection (Table 4). Similarly, the Holm-Sidak multiple comparisons test revealed that Group 4 had a lower cumulative AF-free rate than the other 3 groups $(P<$
0.05 each) (Fig. 1). As for LV hypertrophy among 4 groups, the frequency of LV hypertrophy was highest in Group 4 with $0 \%$ in Group 1, $0 \%$ in Group 2, $16 \%$ in Group 3, and 31\% in Group $4(P<0.01)$.

\section{Discussion}

Our results demonstrated the following major findings. First, among ESUS patients with ICMs implantation, AF was detected in $24 \%$ of patients during observation (median, 442 days) after ICMs implantation. Second, patients with the combination of low LVFS and high E/e' displayed a HR for AF detection about eight times higher than other combinations of LVFS and E/e'. Third, the combination of low LVFS and high E/e' was

\section{Advance Publication Journal of Atherosclerosis and Thrombosis}


independently associated with $\mathrm{AF}$ detection after adjusting for age, BNP, PAC, and LAD. Our results indicate the combination of low LVFS and high E/e' would be the simple and useful tool for stratifying AF risk among ESUS patients with ICMs implantation.

The rate of AF detection in the present study was $24 \%$ during a median of 71 days after implantation. The rate of AF detection in our cohort was higher than that in the CRYSTAL-AF study, despite the relatively shorter observation period ${ }^{1}$. The higher rate of $\mathrm{AF}$ detection in our study may reflect the higher median age of our cohort. A higher proportion of patients underwent transesophageal echocardiography before ICMs implantation, which may also have contributed to stricter diagnosis of ESUS and a resulting higher rate of $\mathrm{AF}$ detection in the present study.

We determined the cutoff value of LVFS (35.5\%) and E/e' (8.65) by ROC analysis. According to the previous studies, the normal value of LVFS was reported as more than $30 \%{ }^{13)}$. As for E/e', definitively normal values of $E / e^{\prime}$ have been reported as $<8.0$ and definitely abnormal values of $E / e^{\prime}$ have been reported as $>14.0$, with E/e' between 8.0 to 14.0 considered indeterminate ${ }^{14}$. In the present study, the IQR of LVFS was $32-41 \%$ and the IQR for E/e' was $7.7-$ 12.7, with most patients showed normal LV systolic and diastolic function and these cutoff values of LVFS and E/e' were not far from the above-normal values.

A recent study has reported that patients with mild LV systolic dysfunction have a higher frequency of AF than those with normal LV systolic function ${ }^{4)}$. LV systolic dysfunction has been observed in patients with LV hypertrophy, which leads to congestion of the left atrium and cause $\mathrm{AF}^{4)}$. The high average $\mathrm{E} / \mathrm{e}^{\text {' }}$ could reflect increased LV filling pressure and LV diastolic dysfunction, which directly leads to $\mathrm{AF}^{3,4)}$. In addition, LV systolic and diastolic dysfunction have also been known as characteristics of LV hypertrophy, which is also associated with a high frequency of $A F^{4,16)}$. Therefore, both characteristics of LV systolic and diastolic dysfunction may reflect the underlying LV remodeling related to $\mathrm{AF}$.

The LV wall thickness and LV systolic dysfunction has been associated with $\mathrm{AF}$ in the previous population-based study ${ }^{6}$. In the present study, the combination of low LVFS and high E/e' was independently associated with $\mathrm{AF}$ detection after adjusting for several factors related to the LA remodeling $^{7-9,17)}$. The combination of low LVFS and high E/e' are representative findings of LV hypertrophy, and may offer surrogates for AF detection in patients with ESUS with ICMs. In other words, patients with AF detected by ICMs may show slight LV remodeling despite the low frequency of AF. The present study might suggest the LV remodeling could serve as the strong marker for AF detection even in ESUS patients. Since both LVFS and E/e' can be calculated from standard transthoracic echocardiography, these parameters may be useful for stratifying the risk of covert AF before ICMs implantation.

There are several limitations in the present study. First, this study was a single-center, retrospective study with a small subject cohort. We therefore could not perform multivariable analyses adjusting for a sufficient number of confounding factors. Second, missing values for E/e' were not rare. Third, our results have yet to be verified in an external validation cohort, which limits the generalizability of our results.

\section{Conclusion}

Patients displaying characteristics of low LVFS and high E/e' were associated with $\mathrm{AF}$ detection among ESUS patients after ICMs implantation. Assessment of LVFS and E/e' using standard transthoracic echocardiography may offer a useful surrogate for AF detection by ICMs in ESUS patients. Further validation studies are warranted.

\section{Acknowledgements}

None.

\section{Notice of Grant Support}

This study was supported in part by a Grant-inAid for Scientific Research from the Japan society for the promotion of science (20K16716).

\section{Conflict of Interests}

Dr. Ikenouchi reports no disclosures.

Dr. Koge reports lecture fees from Medtronic.

Dr. Yamaguchi reports no disclosures.

Dr. Egashira reports no disclosures.

Dr. Tanaka reports no disclosures.

Dr. Washida reports no disclosures.

Dr. Nagase is affiliated with the department endowed by Japan Medtronic Inc.

Dr. Kusano reports honoraria from DaiichiSankyo, Bristol-Myers Squibb, Biotronik Japan, and Medtronic Japan, and research grants from Medtronic Japan.

Dr. Toyoda reports honoraria from DaiichiSankyo, Bayer Yakuhin, Bristol Myers Squibb, Takeda, and Nippon Boehringer Ingelheim, outside the 
submitted work.

Dr. Ihara reports no disclosures.

Dr. Koga reports clinical research funding from Nippon Boehringer Ingelheim.

\section{References}

1) Sanna T, Diener HC, Passman RS, Lazzaro VD, Bernstein RA, Morillo CA, Rymer MM, Thijs V, Rogers T, Beckers $\mathrm{F}$, Lindborg $\mathrm{K}$ and Brachmann J; CRYSTAL AF investiagtors. Cryptogenic stroke and underlying atrial fibrillation. N Engl J Med, 2014; 370: 2478-2486

2) Iwasaki Y, Nishida K, Kato T, Nattel S. Atrial fibrillation pathophysiology. Implications for management. Circulation, 2011; 124: 2264-2274

3) Rosenberg MA, Manning WJ. Diastolic dysfunction and risk of atrial fibrillation. A mechanistic appraisal. Circulation, 2012; 126: 2353-2362

4) Seko Y, Kato T, Haruna T, Izumi T, Miyamoto S, Nakane E, Inoko M. Association between atrial fibrillation, atrial enlargement, and left ventricular geometric remodeling. Sci Rep, 2018; 8: 1-8

5) Patel DA, Lavie CJ, Milani RV, Gilliland Y, Shah S, Ventura HO. Association of left ventricular geometry with left atrial enlargement in patients with preserved ejection fraction. Congest Heart Fail, 2012; 18: 4-8

6) Vaziri SM, Larson MG, Benjamin EJ. Echocardiographic predictors of nonrheumatic atrial fibrillation. The Framingham Heart Study Circulation, 1994; 89: 724-730

7) Yaghi S, Kamel H, Elkind MSV. Atrial cardiopathy: a mechanism of cryptogenic stroke. Expert Rev Cardiovasc Ther, 2017; 15: 591-599

8) Todo K, Iwata T, Doijiri R, Yamagami H, Morimoto M, Hashimoto T, Sonoda K, Yamazaki H, Koge J, Okazaki S, Sasaki T, Mochizuki H. Frequent premature atrial contraction in cryptogenic stroke predict atrial fibrillation detection with insertable cardiac monitor. Cerebrovasc Dis, 2020; $5: 1-7$

9) Cotter PE, Martin PJ, Ring L, Martin PJ, Warburton EA, Belham M, Pugh PJ. Incidence of atrial fibrillation detected by implantable loop recorders in unexplained stroke. Neurology, 2013; 80: 1546-1550
10) Hart RG, Diener HC, Coutts SB, Easton JD, Granger CB, O’Donnell MJ, Sacco RL, Connolly SJ; Cryptogenic stroke/ESUS international working group. Embolic strokes of undetermined source: the case for a new clinical construct. Lancet Neurol, 2014; 13: 429-438

11) Toyoda K, Okumura K, Hashimoto Y, Ikeda T, Komatsu T, Hirano T, Fukuda H, Matsumoto K, Yasaka M. Identification of covert atrial fibrillation in cryptogenic ischemic stroke: current clinical practice in Japan. J Stroke Cerebrovasc Dis, 2016; 25: 1829-1837

12) Matsuo S, Imai E, Horio M, Yasuda Y, Tomita K, Nitta K, Yamagata K, Tomino Y, Yokoyama H, Hishida A; Collaborators developing the Japanese equation for estimated GFR. Revised equations for estimated GFR from serum creatinine in Japan. Am J Kidney Dis, 2009; 53: 982-992

13) Kihara Y, Takenaka K, Hayashi T. Standard measurement of cardiac function indexes. J Med Ultrasonics, 2006; 33: 371-381

14) Nagueh SF, Smiseth OA, Appleton CP, Byrd BF, Dokainish H, Edvardsen T, Flachskampf FA, Gillebert TC, Klein AL, Lancellotti P, Marino P, Oh JK, Popescu BA, Waggoner AD. Recommendations for the evaluation of left ventricular diastolic function by echocardiography: an update from the American society of echocardiography and the European association of cardiovascular imaging. J Am Soc Echocardiogr, 2016; 29: 277-314

15) Lang RM, Badano LP, Mor-Avi V, Afilalo J, Armstrong A, Ernande L, Flachskampf FA, Foster E, Goldstein SA, Kuznetsova T, Lancellotti P, Muraru D, Picard MH, Rietzschel ER, Rudski L, Spencer KT, Tsang W, Voigt JU. Recommendations for cardiac chamber quantification by echocardiography in adults: an update from the American Society of Echocardiography and the European Association of Cardiovascular Imaging. J Am Soc Echocardiogr, 2015; 28: 1-39

16) Lorell BH, Carabello BA. Left ventricular hypertrophy. Pathogenesis, detection, and prognosis. Circulation, 2000; 102: 470-479

17) Casaclang-Verzosa G, Gersh B, Tsang T. Structural and functional remodeling of the left atrium. Clinical and therapeutic implications for atrial fibrillation. J Am Coll Cardiol, 2008; 51: 1-11 